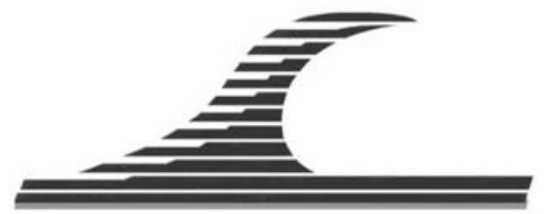

Revue Paralia, Volume 4 (2011) pp 5.1-5.13

Mots-clés : Caractérisation, Valorisation, Sédiments, Dragage,

Mortier, Briques, Tanger, Larache, Maroc.

(C) Editions Paralia CFL

\title{
Caractérisation et valorisation des sédiments de dragage des ports de Tanger et Larache (Maroc)
}

\author{
Laïla BEN ALLAL ${ }^{1}$, Mohammed AMMARI ${ }^{1}$, Ikram FRAR $^{1}$, \\ Amina AZMANI ${ }^{1}$, Nour Eddine BELMOKHTAR ${ }^{1}$
}

\author{
1. Laboratoire de Génie Chimique et Valorisation des Ressources (LGCVR), \\ Université Abdelmalek ESSAADI, Faculté des Sciences et Techniques, \\ Tanger, Maroc. \\ l.benallal@fstt.ac.ma
}

\section{Résumé :}

Le présent travail se place dans une démarche de gestion durable des sédiments de dragage portuaire en étudiant le comportement de ces matériaux pour les valoriser efficacement dans le secteur de la construction, domaine privilégié par cette étude. Une caractérisation complète des sédiments de dragage des ports de Tanger et de Larache a été réalisée à partir d'analyses chimiques, physiques et environnementales. L'étude des propriétés physiques de ces sédiments a révélé que ces sédiments sont généralement des matériaux fins 0/1mm. La caractérisation chimique de ces mêmes sédiments a permis d'évaluer leur potentiel polluant. On a noté l'absence de pollution métallique. La première filière de valorisation de type matériau de construction a concerné les mortiers par substitution du sable par des sédiments de dragage. Les valeurs des résistances à la compression obtenues ont confirmé qu'une substitution partielle du sable par des sédiments de dragage des ports de Tanger et de Larache dans les mortiers est satisfaisante pour un dosage de $20 \%$. La valorisation des sédiments dans la fabrication des briques constitue la deuxième voie de valorisation explorée. La faisabilité technologique de briques renfermant des sédiments de Larache a été démontrée par des essais préliminaires en briqueterie. Pour ces briques, la composition retenue est de $70 \%$ de sédiment. Deux filières de valorisation sont potentiellement envisageables. Ainsi les sédiments de dragage portuaire peuvent constituer une source de matières premières intéressante pour un secteur consommateur de granulats et de matériaux élaborés qu'est le secteur de la construction.

Soumis le 31 octobre 2010, accepté le 4 avril 2011, en ligne le 26 avril 2011.

La seule version examinée est celle écrite en français. La ou les autres versions n'étant pas examinées par le comité de rédaction de la revue, sont donc publiées sous l'entière responsabilité du ou des auteurs.

A TRANSLATED VERSION IN ENGLISH IS AVAILABLE ONLINE

Pour citer cet article :

BEN ALLAL L., AMMARI M., FRAR I., AZMANI A., BELMOKHTAR N.E. (2011). Caractérisation et valorisation des sédiments de dragage des ports de Tanger et Larache (Maroc). Revue Paralia, Vol. 4, pp 5.1-5.13.

DOI:10.5150/revue-paralia.2011.005

(disponible en ligne - http://www.paralia.fr - available online) 


\section{2 : Revue Paralia - Vol. 4 (2011)}

\section{Introduction}

Les sédiments de dragage portuaire sont le produit de l'extraction des sédiments qui se sont déposés sur les fonds notamment des darses portuaires. Ils sont appelés communément vases et sont constitués de phases minérale, organique et liquide. La gestion durable de ces sédiments de dragage demeure aujourd'hui un enjeu important, les volumes des sédiments dragués étant considérables. Au Maroc il est dragué annuellement 3,4 $\mathrm{Mm}^{3}$.

La présente étude s'intéresse à la valorisation des sédiments de dragage bruts des ports de Tanger et de Larache dans les matériaux de construction tels que les mortiers et les briques. Elle s'inscrit bien dans une démarche de gestion globale des sédiments de dragage portuaire qui nécessite la connaissance des sédiments, en quelque sorte leur fiche d'identité (LEVACHER et al., 2011). Pour ce faire, il est au préalable indispensable de déterminer les différentes propriétés des sédiments et ainsi, effectuer leur caractérisation physique, chimique, minéralogique et environnementale. On s’intéresse dans le cadre de cette étude aux sédiments qui proviennent de deux ports du nord du Maroc: le port de Tanger et le port de Larache. Ces deux sédiments ont été incorporés à des mortiers par substitution partielle du sable normalisé et du ciment. Les résistances mécaniques on été mesurées et comparées aux résistances d'un mortier témoin.

\section{Connaissance des sédiments}

\subsection{Présentation des sites de prélèvement}

Les sédiments étudiés ont été prélevés dans les ports de Tanger et de Larache (figure 1). Grâce à sa position stratégique entre l'Atlantique et la Méditerranée, le port de Tanger est le premier port marocain dans le trafic des passagers et du transit international routier.
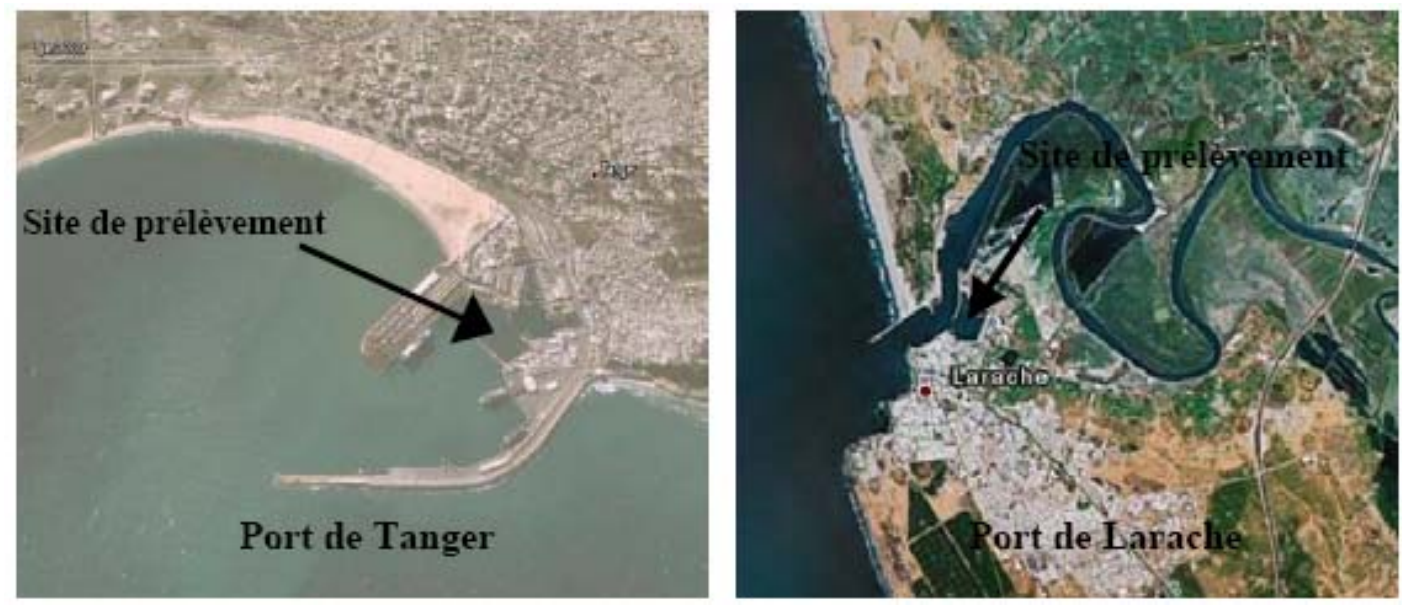

Figure 1. Sites de prélèvement des sédiments. 
Quant au port de Larache, il est situé sur la côte atlantique nord du Maroc à $90 \mathrm{~km}$ au sud de Tanger. C'est un port fluvial installé dans une darse sur la rive gauche de l'oued de Loukkos à $750 \mathrm{~m}$ environ de son embouchure. Le port de Larache se consacre aujourd'hui exclusivement aux activités de pêche.

\subsection{Caractérisation des sédiments bruts}

Elle comporte l'étude des propriétés physiques (paramètre d'état et de nature, teneurs en eau, distributions granulaires, minéralogie), des propriétés chimiques ( $\mathrm{pH}$, conductivité, teneur en carbonates, teneurs en matières organiques et hydrocarbures, teneur en sulfates, teneurs en éléments traces et éléments majeurs) et des caractéristiques environnementales (essais de lixiviation). Les méthodes et matériels de détermination de ces grandeurs sont décrits et les résultats présentés. S'ensuit une discussion sur l'ensemble des résultats de caractérisation qui constituent en soi la fiche d'identité des sédiments. Cette fiche d'identité permettra de connaître l'impact de ces sédiments sur l'environnement et la proposition de méthodes de valorisation adéquates.

\subsubsection{Caractérisation physique}

Tout d'abord, les masses volumiques absolue et apparente des deux sédiments ont été déterminés selon la norme NF P18-555. Les résultats sont rassemblés dans le tableau 1 L'analyse granulométrique a été réalisée par voie humide sur un tamis de $63 \mu \mathrm{m}$ dans le but de séparer la fraction fine et la fraction grossière $(>63 \mu \mathrm{m})$. Les résultats sont présentés dans le tableau 1. Pour la partie grossière qui correspond au refus, elle a été séchée à l'étuve à $110{ }^{\circ} \mathrm{C}$ jusqu'à l'obtention d'une masse constante et tamisée par l'utilisation d'une série de tamis selon la norme NF P 18-560.

Ce sont deux sédiments fins, cependant sur la figure 2, on observe clairement que le sédiment du port de Larache est beaucoup plus fin que le sédiment du port de Tanger.

Tableau 1. Caractéristiques physiques et chimiques des sédiments étudiés.

\begin{tabular}{lll}
\hline Sédiment & Larache & Tanger \\
\hline Masse volumique absolue $\left(\mathrm{g} / \mathrm{cm}^{3}\right)$ & 2,20 & 2,28 \\
Masse volumique apparente $\left(\mathrm{g} / \mathrm{cm}^{3}\right)$ & 1,53 & 1,30 \\
Fraction $<63 \mu \mathrm{m}(\%)$ & 94 & 41 \\
Fraction $>63 \mu \mathrm{m}(\%)$ & 6 & 59 \\
Siccité à $40^{\circ} \mathrm{C}(\%)$ & 49,4 & 58,6 \\
$\mathrm{pH}$ & 7,8 & 8,0 \\
Conductivité $\left(\mathrm{mS} / \mathrm{cm}^{2}\right)$ & 6,5 & 6,6 \\
Teneur en carbonates $(\%)$ & 8,0 & 10,5 \\
Matière organique $(\%)$ & 4,2 & 7,0 \\
Hydrocarbures $(\%)$ & 1,3 & 5,1 \\
Sulfates (\%) & 0,7 & 1,1 \\
\hline
\end{tabular}




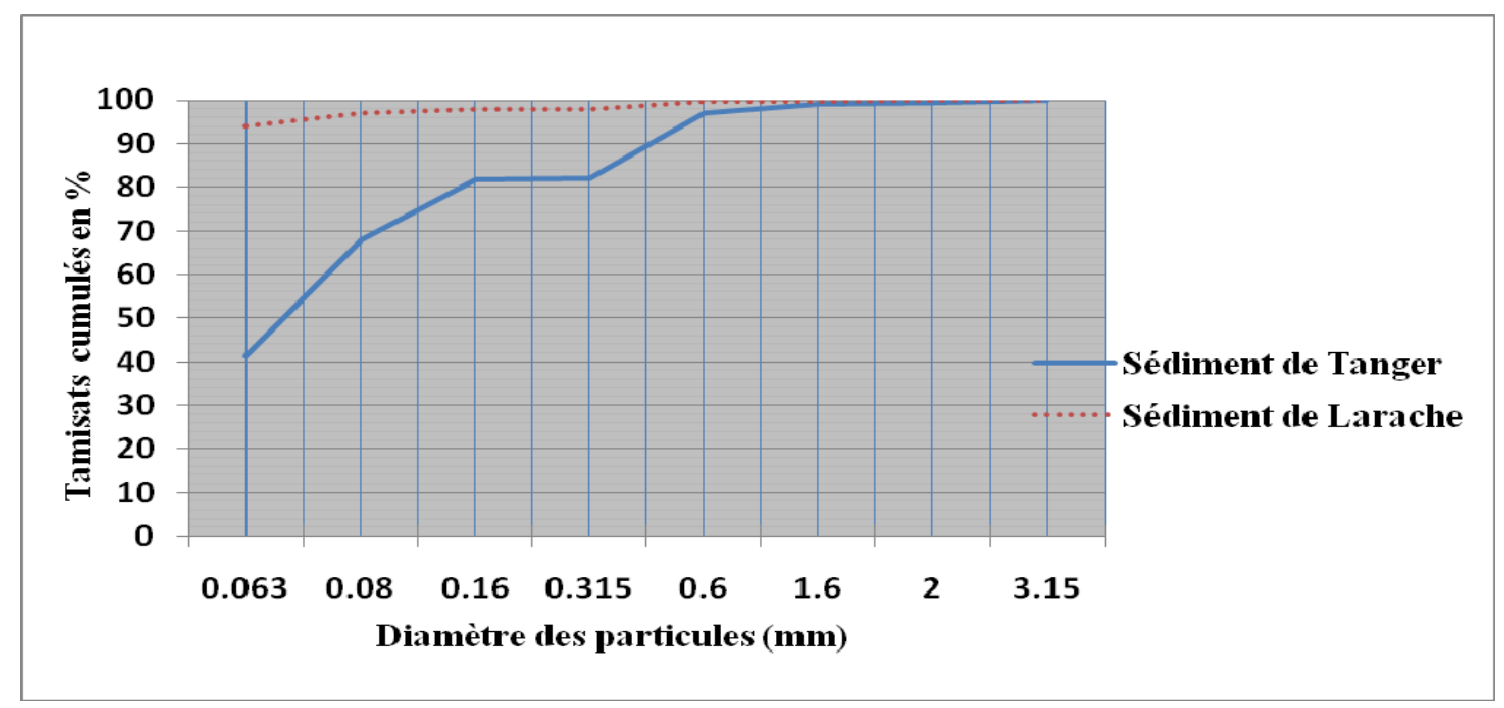

Figure 2. Courbes granulométriques des sédiments.

On a procédé à la mesure de la siccité des sédiments à $40^{\circ} \mathrm{C}$. Le principe repose sur le séchage de l'échantillon dans une étuve à $40{ }^{\circ} \mathrm{C}$ jusqu'à l'obtention d'une masse constante selon la norme NF ISO 11464. Les mesures relatives aux sédiments (valeurs moyennes sur 3 échantillons) sont consignées dans le tableau 1. Ces valeurs dépendent fortement des conditions de dragage.

Cette élimination d'humidité entre dans le cas de la préparation des échantillons pour les analyses physico-chimiques. La température est fixée à $40{ }^{\circ} \mathrm{C}$ pour éviter toute altération de la matière organique (KRIBI, 2005).

Une analyse minéralogique des sédiments étudiés a été effectuée à partir d'analyses qualitatives par DRX qui ont été réalisées sur une fraction broyée de l'ordre de $80 \mu \mathrm{m}$ pour les deux sédiments à l'aide d'un appareil Spinner PW 3064. Il en résulte que la calcite et le quartz prédominent.

\subsubsection{Caractérisation chimique}

Elle comprend quelques propriétés chimiques utiles au choix des procédés de valorisation (valorisation dans les matériaux cimentaires ou céramiques) et la détermination de certaines teneurs en constituants lesquels de par leur présence peuvent contribuer ou perturber la mise en œuvre de tel ou tel procédé.

La détermination du $\mathrm{pH}$ est un indicateur dans le cas des traitements aux liants hydrauliques de sédiments. Un $\mathrm{pH}$ acide entraîne la mise en solution des sels métalliques, la désorption des cations et l'adsorption des anions (LIONS, 2004). Lors de l'utilisation d'un liant hydraulique, les cations métalliques précipitent sous forme d'hydroxydes métalliques peu à pas solubles en raison de la forte alcalinité de la matrice cimentaire (CONNER, 1990). 
La valeur du pH exprime l'acidité réelle et prend en compte les ions $\mathrm{H}_{3} \mathrm{O}^{+}$libres dans la phase liquide.

Les mesures de $\mathrm{pH}$ des deux sédiments ont été déterminées selon la norme NF X 31103, voir tableau 1.

Une autre propriété évaluée est la conductivité électrique qui constitue une bonne appréciation des concentrations globales des matières en solution dans les sédiments.

La norme utilisée est NF ISO 11265. Les valeurs moyennes mesurées de la conductivité sont dans le tableau 1.

L'évaluation des différentes teneurs en constituants concerne d'abord les carbonates, les matières organiques et les sulfates. Les compositions chimiques sont obtenues à partir de dosages effectués en métaux lourds et éléments majeurs. La stabilisation aux liants hydrauliques est une des méthodes les plus utilisées pour stabiliser et solidifier les déchets riches en métaux lourds tels que les sédiments. Une présence importante de matière organique peut influencer l'hydratation du ciment et réduire les résistances.

La détermination des teneurs en carbonates (voir tableau 1) a été réalisée à l'aide du calcimètre de Bernard. Cette mesure est décrite par la norme NF ISO 10693.

Puis la mesure de la teneur en matière organique MO a été effectuée en utilisant la méthode d'oxydation sulfochromique. Le principe de cet essai repose sur l'oxydation de la matière organique par le bichromate de potassium puis le titrage de l'excès du bichromate par le sel de Mohr selon la norme : NF ISO 14235. Les valeurs moyennes déduites sont dans le tableau 1.

La présence potentielle d'hydrocarbures HAP a justifié la mesure de la teneur en hydrocarbures. Les hydrocarbures comme toute matière organique sont solubles dans des solvants organiques. Le principe de cet essai repose sur l'extraction des hydrocarbures présents dans les sédiments par un solvant organique, le toluène a été utilisé. Les valeurs (tableau 1) indiquent bien la présence d'HAP pour le port de Tanger. Le dosage des sulfates a été préconisé dans la mesure où les sulfates sont des éléments perturbateurs dans les procédés de traitements aux liants hydrauliques. Les sulfates sont des composés nécessaires pour réguler la prise des ciments. Cependant, si la teneur devient trop importante, des dégradations du matériau traité par des liants hydrauliques peuvent apparaître (gonflements importants, développement d'une fissuration importante, une diminution des résistances mécaniques), (BARYLA et al., 2000). Les sulfates ont été dosés dans les deux sédiments par gravimétrie. Les valeurs sont consignées dans le tableau 1.

Afin d'avoir une idée plus précise sur la composition chimique des sédiments étudiés, on a procédé aux dosages des métaux lourds et des éléments majeurs. Les éléments majeurs ont été dosés par spectrométrie d'absorption atomique et les éléments traces (métaux lourds) ont été dosés par spectrométrie de masse couplée à un plasma inductif (ICP-MS). Les résultats obtenus sont rassemblés dans les tableaux 2 et 3. 
Tableau 2. Composition chimique en éléments majeurs dans les sédiments en \%.

\begin{tabular}{|c|c|c|c|c|c|c|c|c|}
\hline $\begin{array}{l}\text { Eléments } \\
\text { majeurs }\end{array}$ & $\mathrm{SiO}_{2}$ & $\mathrm{Al}_{2} \mathrm{O}_{3}$ & $F \boldsymbol{e}_{2} \mathrm{O}_{3}$ & $\mathrm{CaO}$ & $M g O$ & $\mathrm{SO}_{3}$ & $\mathrm{~K}_{2} \mathrm{O}$ & $\mathrm{Na}_{2} \mathrm{O}$ \\
\hline $\begin{array}{l}\text { Sédiment de } \\
\text { Larache }\end{array}$ & 49,6 & 14,5 & 9,1 & 11,2 & 0,5 & 0,6 & 1,7 & 2,0 \\
\hline $\begin{array}{l}\text { Sédiment de } \\
\text { Tanger }\end{array}$ & 48,5 & 11,9 & 7,4 & 11,7 & 1,2 & 1,1 & 1,7 & 2,0 \\
\hline
\end{tabular}

Tableau 3.Concentrations des éléments traces des sédiments en $\mathrm{mg} / \mathrm{kg}$ de sédiment sec.

\begin{tabular}{lll}
\hline Eléments traces & $\begin{array}{l}\text { Sédiment de Larache } \\
(\mathbf{m g} / \mathbf{k g})\end{array}$ & $\begin{array}{l}\text { Sédiment de Tanger } \\
(\mathbf{m g} / \mathbf{k g})\end{array}$ \\
\hline Chrome (Cr) & 0,22 & 0,10 \\
Arsenic $(\mathrm{As})$ & 0,02 & 0,01 \\
Nickel $(\mathrm{Ni})$ & 0,06 & 0,07 \\
Zinc $(\mathrm{Zn})$ & 0,01 & 0,39 \\
Cuivre $(\mathrm{Cu})$ & 0,08 & 0,38 \\
\hline
\end{tabular}

Tableau 4. Niveaux relatifs aux éléments traces (en $\mathrm{mg} / \mathrm{kg}$ de sédiment sec analysé sur la fraction inférieure à $2 \mathrm{~mm}$ ), GEODE (2000).

\begin{tabular}{lll}
\hline Éléments traces & Niveau $\boldsymbol{N}_{\mathbf{1}}$ & Niveau $\mathrm{N}_{\mathbf{2}}$ \\
\hline Arsenic $(\mathrm{As})$ & 25 & 50 \\
Chrome $(\mathrm{Cr})$ & 90 & 180 \\
Cuivre $(\mathrm{Cu})$ & 45 & 90 \\
Nickel $(\mathrm{Ni})$ & 37 & 74 \\
Zinc $(\mathrm{Zn})$ & 276 & 552 \\
\hline
\end{tabular}

\subsubsection{Précisions sur la définition des niveaux $N_{1}$ et $N_{2}$}

Pour le niveau $\mathrm{N}_{1}$, au-dessous duquel les opérations de dragage et d'immersion seraient autorisées sans autres études, l'impact potentiel est jugé neutre ou négligeable, voir tableau 4. Les valeurs observées se révélant comparables aux "bruits de fond" environnementaux.

Pour le niveau $\mathrm{N}_{2}$, au-dessus duquel les opérations d'immersion seraient susceptibles d'être interdites sous réserve que cette interdiction soit la solution de gestion la moins dommageable pour l'environnement, voir tableau 4. Une investigation complémentaire est généralement nécessaire car des indices peuvent laisser présager un impact potentiel de l'opération. Une étude d'impact approfondie est alors jugée indispensable.

Entre les niveaux $\mathrm{N}_{1}$ et $\mathrm{N}_{2}$, une investigation complémentaire peut s'avérer nécessaire en fonction du projet considéré et du degré de dépassement du niveau $\mathrm{N}_{1}$. Des tests sont alors pratiqués pour évaluer la toxicité globale des sédiments. 


\subsubsection{Etude environnementale : lixiviation des sédiments bruts}

Des essais de lixiviation sont réalisés selon la norme européenne NF EN 12457. Les échantillons sont en contact avec de l'eau distillée sous agitation pendant $24 \mathrm{~h}$. Le rapport liquide/solide est de 10 . Ils permettent d'obtenir les dosages concernant les chlorures et les sulfates.

Les chlorures sont dosés par titrage par le nitrate d'argent. Les résultats sont rassemblés dans le tableau 5.

Pour les sulfates, ils sont dosés par spectrophotométrie UV. Les résultats sont rassemblés dans le tableau 5.

Tableau 5. Teneur en chlorures et en sulfates $(\mathrm{mg} / \mathrm{kg})$.

\begin{tabular}{lll}
\hline Sédiment & Larache & Tanger \\
\hline Teneur moyenne en chlorures $(\mathrm{mg} / \mathrm{kg})$ & 940 & 1020 \\
\hline Teneur moyenne en sulfates $(\mathrm{mg} / \mathrm{kg})$ & 885 & 1268 \\
\hline
\end{tabular}

\subsubsection{Discussion et interprétation des résultats}

La comparaison des concentrations en éléments traces des sédiments de dragage étudiés aux niveaux de référence GEODE (2000), (tableau 4) permet de conclure à une absence de pollution métallique.

La teneur en matières organiques pour le sédiment de dragage de Tanger est plus élevée que celle du sédiment de Larache.

Ce résultat peut s'expliquer par l'intensité des rejets urbains dans le port de Tanger. Le dosage des hydrocarbures montre un taux élevé pour le sédiment du port de Tanger, ceci en raison de la nature de la grande activité de ce port.

Tableau 6. Seuils de concentrations respectifs des polluants pour les différentes classes de déchets (CUE, 2003).

\begin{tabular}{|c|c|c|c|c|c|c|}
\hline Paramètres & $\begin{array}{l}\text { Classe III } \\
\text { Stockage } \\
\text { de déchets } \\
\text { inertes }\end{array}$ & $\begin{array}{l}\begin{array}{l}\text { Valeurs } \\
\text { seuils } \\
\text { (mg/kg de } \\
\text { matière } \\
\text { sèche) }\end{array} \\
\end{array}$ & $\begin{array}{l}\text { Classe II } \\
\text { Stockage } \\
\text { de déchets } \\
\text { non } \\
\text { dangereux } \\
\end{array}$ & 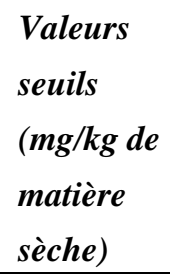 & $\begin{array}{l}\text { Classe I } \\
\text { Stockage } \\
\text { de déchets } \\
\text { dangereux }\end{array}$ & $\begin{array}{l}\begin{array}{l}\text { Valeurs } \\
\text { seuils } \\
\text { (mg/kg de } \\
\text { matière } \\
\text { sèche) }\end{array} \\
\end{array}$ \\
\hline \multicolumn{7}{|c|}{ SUR MATERIAU BRUT } \\
\hline COT & $<$ & 30000 & $<-<$ & 50000 & $<-<$ & 60000 \\
\hline BTEX & $<$ & 6 & - & - & - & - \\
\hline$P C B$ & $<$ & 1 & - & - & - & - \\
\hline Hydrocarbures & $<$ & 500 & - & - & - & - \\
\hline HAP & $<$ & 50 & - & - & - & - \\
\hline
\end{tabular}




\section{8 : Revue Paralia - Vol. 4 (2011)}

On peut conclure que les deux sédiments sont pollués en hydrocarbures si on compare les taux obtenus aux valeurs seuils autorisées dans les centres de stockage de déchets (CUE, 2003), (tableau 6).

Les teneurs en sulfates (dans la phase solide) obtenues par absorption atomique sont presque égales aux valeurs obtenues par gravimétrie, elles sont respectivement de 0,6 et 0,7\% pour le sédiment de Larache et de même valeur 1,1\% pour le sédiment de Tanger.

La granulométrie du sédiment de Larache montre qu’il est limoneux (cette fraction représente 94\%) alors que le sédiment de Tanger a un caractère sableux légèrement limoneux.

\section{Valorisation en matériaux de construction des sédiments de dragage}

\subsection{Valorisation des sédiments}

La plupart des études ont montré la faisabilité de la substitution de matériaux naturels par des déchets ou sous-produits et la relative bonne qualité des matériaux obtenus (AUBERT, 2002 ; BOUCARD, 2006). En s’inspirant de ces travaux, nous avons choisi d'étudier le comportement des sédiments dans les mortiers en substituant partiellement d'une part le sable normalisé et d'autre part le ciment.

\subsubsection{Substitution de 20\% de sable normalisé par les sédiments}

L'étude menée a pour objectif de mettre en évidence l'influence de la substitution partielle du sable par les sédiments bruts sur le comportement en résistance des mortiers. Le ciment utilisé est un ciment marocain CPJ 45, avec un ratio eau sur ciment (E/C) de 0,5. Les dosages choisis sont de $1350 \mathrm{~kg}$ de sable, pour $450 \mathrm{~kg}$ de ciment.

Ainsi des éprouvettes de $4 \times 4 \times 16 \mathrm{~cm}$ ont été confectionnées selon la norme EN 196-1 puis testées. Les résistances en compression $\mathrm{R}_{\mathrm{cj}}$ et en flexion $\mathrm{R}_{\mathrm{tfj}}$ ont été mesurées à 7 jours et 28 jours. Les valeurs de référence sont celles obtenues pour un mortier normal. L’ensemble des valeurs moyennes des résistances sont récapitulées dans le tableau 7.

Tableau 7. Résistances en compression et en traction par flexion des mortiers pour une substitution de $20 \%$ de sable normalisé.

\begin{tabular}{lllll}
\hline $\boldsymbol{R}_{\boldsymbol{c 7}}(\mathbf{M P a})$ & $\boldsymbol{R}_{\boldsymbol{c 2 8}}(\mathbf{M P a})$ & $\boldsymbol{R}_{\boldsymbol{t} \mathbf{7}}(\mathbf{M P a})$ & $\boldsymbol{R}_{\mathbf{t} 28 \mathbf{( M P a})}$ & Référence des mortiers \\
\hline 32,0 & 45,0 & 5,6 & 6,4 & Mortier normal CPJ 45 \\
18,0 & 28,5 & 3,9 & 5,8 & Mortier sédiment de Tanger \\
19,3 & 30,3 & 4,1 & 6,0 & Mortier sédiment de Larache \\
\hline
\end{tabular}

\subsubsection{Substitution de 20\% de ciment CPJ 45 par les sédiments}

Dans cette partie, les mortiers ont été confectionnés par substitution de $20 \%$ de ciment CPJ 45 par les sédiments. Le ratio eau sur liant i.e. ciment et sédiment, (E/L) est de 0,5. 
Comme précédemment, les résistances à la compression $\mathrm{R}_{\mathrm{cj}}$ et à la flexion $\mathrm{R}_{\mathrm{tfj}}$ ont été mesurées sur des éprouvettes $4 \times 4 \times 16 \mathrm{~cm}$ et testées à 7 et 28 jours. Les résultats sont rassemblés dans le tableau 8.

Tableau 8. Résistances en compression et en traction par flexion des mortiers pour une substitution de $20 \%$ de ciment.

\begin{tabular}{lllll}
\hline $\boldsymbol{R}_{\boldsymbol{c 7}}(\mathbf{M P a})$ & $\boldsymbol{R}_{\boldsymbol{c 2 8}}(\mathbf{M P a})$ & $\boldsymbol{R}_{\boldsymbol{t f 7}}(\mathbf{M P a})$ & $\boldsymbol{R}_{\mathbf{t} 28 \mathbf{( M P a})}$ & Référence des mortiers \\
\hline 32 & 45 & 5,6 & 6,4 & Mortier normal CPJ 45 \\
14,2 & 23,4 & 3,3 & 5,4 & Mortier sédiment de Tanger \\
14,2 & 24,6 & 3,4 & 5,5 & Mortier sédiment de Larache \\
\hline
\end{tabular}

\subsubsection{Discussion et interprétation des résultats}

Le sédiment du port de Larache donne un mortier plus résistant que le sédiment du port de Tanger lorsque les deux sédiments bruts remplacent 20\% du sable normalisé. Ce résultat peut s'expliquer par le taux élevé de la matière organique présent dans le sédiment du port de Tanger. D’après KUJALA et al. (1996), la présence élevée de la matière organique peut empêcher la bonne hydratation de la pâte de ciment. Les matières organiques capteraient les ions calcium libérés par le ciment pendant la phase d'hydratation et ne permettraient plus de former les hydrates nécessaires à la fabrication des liens forts entre les particules. La diminution du caractère résistant peut être provoquée par la présence de matières organiques à caractère inhibiteur. Selon (FANTOZZI-MERLE, 2003), la présence de polluants organiques peut aussi affecter la résistance mécanique.

Dans le cas de la substitution du ciment par 20\% de sédiment, les résistances obtenues pour les deux mortiers sont comparables. Mais ces résistances restent très inférieures aux résistances du mortier de référence. Le résultat peut s'expliquer par la diminution de la quantité de ciment. Une diminution de résistance est incontournable.

Le modèle prédictif de Bolomey permet de confirmer les résultats expérimentaux obtenus : $\mathrm{f}_{\mathrm{cj}}=\mathrm{k}_{\mathrm{b}} \mathrm{f}_{\mathrm{mj}}[\mathrm{C} /(\mathrm{E}+\mathrm{V})-0,5]$

$\mathrm{f}_{\mathrm{cj}}=$ résistance en $(\mathrm{MPa})$ du mortier à j jours.

$\mathrm{k}_{\mathrm{b}}=$ coefficient granulaire qui dépend du granulat utilisé (on prend $\mathrm{k}_{\mathrm{b}}=0,50$ ).

$\mathrm{f}_{\mathrm{mj}}=$ résistance vraie du ciment à j jours, mesurée sur mortier normal (EN 196-1).

C, E, V : masses volumiques respectifs de ciment, d'eau et d'air $\left(\mathrm{kg} / \mathrm{m}^{3}\right)$.

Nous avons déterminé les résistances théoriques en compression :

Au bout de 7 jours $\mathrm{f}_{\mathrm{C} 7}=15,3 \mathrm{MPa}$.

Au bout de 28 jours $\mathrm{f}_{\mathrm{c} 28}=21,5 \mathrm{MPa}$.

Donc globalement, le remplacement partiel du ciment par les deux sédiments conduit aux variations de résistances prévues par le modèle de Bolomey. 
3.2 Valorisation des sédiments dans l'élaboration de briques : étape de faisabilité

La composition chimique (\% en éléments majeurs) des deux sédiments bruts et la composition chimique des argiles utilisées pour la fabrication des produits de terre cuite sont comparables (ALVISET, 1994). Cette analogie de composition a orienté nos recherches vers la valorisation des sédiments comme matière première dans les briques. Il s’agit d'une étude de faisabilité pour ce type de valorisation. Les sédiments ont été utilisés dans la fabrication de briques par substitution partielle de l'argile entrant dans la formulation. La confection des briques a été réalisée au sein même de la briqueterie $\mathrm{Al}$ Andalous de Tanger.

La préparation des échantillons de briques consiste en un mélange de $70 \%$ de sédiments et 30\% d’argile. Après séchage à l’air libre, les trois matériaux (sédiment de Larache, sédiment de Tanger et argile) sont broyés puis tamisés à $1 \mathrm{~mm}$. Après malaxage, moulage et séchage de la pâte, les briques-éprouvettes sont séchées à une température de cuisson de $920{ }^{\circ} \mathrm{C}$ pendant 32 heures. Les premiers constats sont encourageants puisque la fabrication de briques s'est révélée possible. Les photos de la figure 3 montrent l'aspect des briques obtenues en substituant 70\% de l'argile utilisée par les sédiments de dragage.

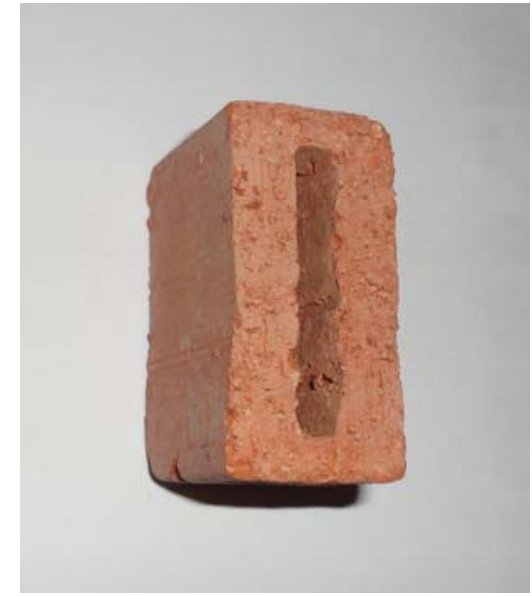

Sédiment de Larache

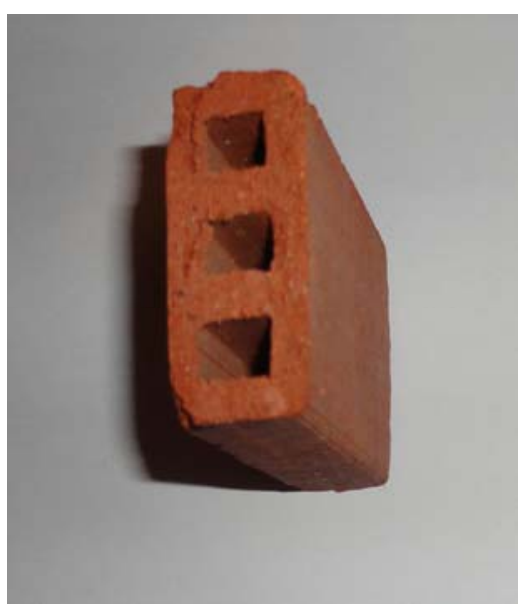

Sédiment de Tanger

Figure 3. Briques fabriquées avec un taux de substitution de $70 \%$ de sédiments, de mêmes dimensions $2,5 \times 5,0 \times 7,5 \mathrm{~cm}$.

Un premier constat s'impose pour cette étape de faisabilité. La texture et l'aspect des briques obtenues, sont comparables à la brique de référence de la société. L’idée de valoriser les sédiments étudiés dans la fabrication de briques paraît envisageable. A ce stade, il convient de vérifier les performances mécaniques et environnementales de ces briques incorporant des sédiments. 


\section{Conclusions}

La caractérisation physique des sédiments de dragage des ports de Tanger et Larache a révélé que ces sédiments sont des matériaux fins à très fins. Leurs principaux constituants sont des limons et des sables fins du point de vue granulométrique. La caractérisation chimique des sédiments a permis d'évaluer leur potentiel polluant. On a surtout noté l'absence de pollution métallique. Cependant, le sédiment de dragage du port de Tanger présente un taux élevé d’hydrocarbures en raison de la nature de l'activité du port.

L’impact sur l'environnement, évalué au travers d'essais de lixiviation, montre une teneur modérée en chlorures et en sulfates dans les deux sédiments comparativement aux valeurs seuils d'acceptation dans les centres de stockage.

Il s'avère que ces sédiments à granulométrie fine constituent des matériaux économiques locaux attractifs pour les entreprises marocaines de la construction notamment les briqueteries.

Les valeurs des résistances à la compression des mortiers ont montré que la substitution partielle du sable par les sédiments de dragage des ports de Tanger et de Larache dans les mortiers est satisfaisante mais avec un taux de réduction de résistance de l'ordre de $30 \%$. Avec de telles résistances, les utilisations potentielles concernent les mortiers d'enduit ou de maçonnerie, les blocs manufacturés. Pour ces produits, on peut accepter un remplacement partiel du sable normalisé par ces sédiments.

Les sédiments en remplacement partiel du ciment conduisent à une chute de résistance de 45\%. Une partie de cette de baisse de résistance est expliquée par le fait que la quantité de ciment a été diminuée de $20 \%$ : une discussion basée sur une analyse par les lois de Bolomey ou de Féret avait été proposée. D’après SEMCHA (2006), une activation thermique des sédiments de dragage (à grande teneur en fines) pourrait améliorer les propriétés pouzzolaniques et la performance mécanique à longue échéance. Le traitement thermique transforme la composition minéralogique du matériau et la combinaison de nouvelles structures avec la chaux aboutissent à la formation de liant hydraulique.

La valorisation des sédiments dans les briques constitue une étape de faisabilité qui est prometteuse. Cette filière "briques" est plus que potentielle et des travaux pour optimiser la fabrication sont à poursuivre.

Enfin, on peut entrevoir que ces deux sédiments de dragage portuaire peuvent constituer une source de matières premières locale très intéressante, notamment pour le secteur de la construction fort consommateur de granulats.

\section{Remerciements}

Les auteurs expriment leurs sincères remerciements à A. Ghayati et M. Allouch respectivement directeur et responsable de production de la briqueterie Al Andalous de Tanger. 


\section{Références bibliographiques}

ALVISET L. (1994). Matériaux de terre cuite. Techniques de l’ingénieur. 10 Mai 1994. AUBERT J.E. (2002). Valorisation d'une cendre d'incinérateur d'ordures ménagères traitée par le procédé REVSOL ${ }^{\circledR}$ dans les bétons hydrauliques. Thèse de doctorat. Université Paul Sabatier de Toulouse.

BARYLA J.M., CHENAIS V., GAVOIS L., HAVARD H. (2000). Effet de sulfates et sulfures sur des marnes traitées à la chaux et au liant routier sur un chantier autoroutier. Bulletin des laboratoires des Ponts Chaussées n²24, pp 39-48.

BOUCARD L. (2006). Traitement et valorisation des sédiments fluviaux. Thèse de doctorat. Université des sciences et techniques de Lille.

CUE -Conseil de l’Union Européenne- (2003). 2003/33/CE : Décision du conseil du 19 décembre 2002 établissant des critères et des procédures d'admission des déchets dans les décharges, conformément à l'article 16 et à l'annexe II de la directive 1999/31/CE. Journal Officiel de l’Union Européenne n L011 du 16/01/2003.

EN 196-1 (2006). Méthodes d'essais des ciments. Détermination des résistances mécaniques. Avril 2006.

CONNER J.R. (1990). Chemical fixation and solidification of hazardous wastes. Van Nostrand Reinhold, New York. 692 p.

FANTOZZI-MERLE C. (2003). Etude des matériaux à base de liant hydraulique contenant des polluants organiques modèles: propriétés structurales et de transfert. Thèse, INSA de Lyon.

GEODE (2000). Arrêté du 14 Juin 2000 J.O du 10 Août 2000 relatif aux niveaux de référence à prendre en compte lors d'une analyse de sédiments marins ou estuariens présents en milieu naturel ou portuaire.

KRIBI S. (2005). Décomposition des matières organiques et stabilisation des métaux lourds dans les sédiments de dragage. Thèse de doctorat. Institut national des sciences appliquées de Lyon.

KUJALA K., MÄKIKYRÖ M., LEHTO O. (1996). Effect of humus on the binding reaction in stabilized soils. IS - Tokyo’96. Second International Conference on Ground Improvement Geosystems - Grouting and Deep Mixing. May 14-17, 1996.

LEVACHER D., SANCHEZ M., DUAN Z., LIANG Y. (2011). Valorisation en unité pilote de sédiments méditerranéens : Etude des caractéristiques géotechniques et de la perméabilité, Revue Paralia, Vol. 4, pp 4.1-4.20. doi:10.5150/revue-paralia.2011.004

LIONS J. (2004). Etude hydrogéochimique de la mobilité de polluants inorganiques dans des sédiments de curage mis en dépôt: Expérimentations, étude in situ et modélisation. Thèse de doctorat. Ecole Nationale supérieure des Mines de Paris.

NF EN 12457 (2002). Caractérisation des déchets. Lixiviation. Essai de conformité pour la lixiviation des déchets fragmentés et des boues. Décembre 2002

NF ISO 11464 (1994). Prétraitement des échantillons pour analyses physico-chimiques. Décembre 1994. 
NF ISO 10693 (1995). Détermination de la teneur en carbonates. Méthode volumétrique (Indice de classement : X31-105). Juin 1995.

NF ISO 11265 (1995). Détermination de la conductivité électrique spécifique. Janvier 1995.

NF ISO 14235 (1998). Qualité du sol. Dosage du carbone organique par oxydation sulfochromique. Septembre 1998.

NF P 18-560 (1990). Analyse granulométrique par tamisage. Septembre 1990.

NF P 18-555 (1990) Mesure des masses volumiques, coefficient d'absorption et teneur en eau des sables. Décembre 1990.

NF X 31-103 (1988). Détermination du pH dans l'eau.

SEMCHA A (2006).Valorisation des sédiments de dragage. Application dans le BTP, cas du barrage de Fergoug. Thèse de doctorat. Université de Reims ChampagneArdenne. 
5.14 : Revue Paralia - Vol. 4 (2011) 